\title{
Evolution of a Mammalian Promoter Through Changes in Patterns of Transcription Factor Binding
}

\author{
Nandita Singh, Franklin G. Berger \\ Department of Biological Sciences, University of South Carolina, Columbia, SC 29208, USA
}

Re: J Mol Evol (1998) 46(6) 639-648. The legends to Figs. 3-5 of this article were mistakenly omitted from the published version. They appear correctly here.

Fig. 3. Competitive mobility shift analysis of factor binding to site C. A labeled oligonucleotide corresponding to site $\mathrm{C}$ was used as a probe to measure binding of proteins from a liver nuclear extract. Increasing amounts of an unlabeled oligonucleotide representing site $\mathrm{C}$ from $M$. domesticus $(\mathbf{\square})$, M. pahari $(\diamond)$, mutant $\mathrm{B}(\boldsymbol{\Delta})$, and mutant $\Delta \mathrm{B} 3(\times)$ were added to the reaction mixtures, and tested for their ability to inhibit complex formation. The \% binding, representing the relative amount of radioactivity in the complex, was plotted as a function of the amount of competitor.

Fig. 4. Impact of mutations in site B on DNase I footprinting patterns. Fragments containing nucleotides $-378 /-123$ were labeled at nucleotide -378 , and used as probes. Patterns are shown for mutants B4 (lanes 1,2), $\Delta \mathrm{B} 4$ (lanes 3,4), B5 (lanes 5,6), $\Delta \mathrm{B} 5$ (lanes 7,8), and P1 (lanes 9-11). For each fragment, the first lane shows the pattern in the absence of nuclear extract protein, while the second lane shows the pattern in the presence of extract protein. Lane 11 show the results of addition of a cold competitor oligonucleotide corresponding to a consensus NF-I binding site. Brackets indicate binding sites A, B, and $\mathrm{C}$.

Fig. 5. Constructs for transient transfection. All plasmids were derived from pBLCAT2, which contains a CAT reporter gene driven by a minimal $t k$ promoter (see Materials and Methods). The $-365 /-226$ regions from $M$. domesticus (DOM), M. pahari (PAH), and mutants $\Delta \mathrm{B} 5, \mathrm{~B} 5$, and $\mathrm{P} 1$ are shown in the normal orientation relative to the transcriptional start-site (indicated by a bent arrow). Dashed lines indicate the promoter regions; the locations of single base substitutions are denoted by X's; deletions are indicated by parentheses. Open boxes represent the coding region of the $C A T$ reporter; cross-hatched boxes represent the $t k$ promoter. Sites $\mathrm{A}, \mathrm{B}$, and $\mathrm{C}$ are overlined. 